\title{
Office fédéral de la statistique: non!
}

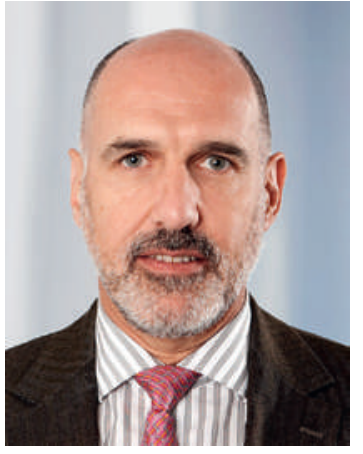

Comme on l'apprendra plus en détail en page 1115 de cette édition du Bulletin des médecins suisses, l'Office fédéral de la statistique (OFS) se lance dans une collecte frénétique de données sur l'activité des médecins et autres soignants en Suisse.

Pas de base légale à cela? Mais voyons, aucun problème! L'intérêt supérieur des banques de données de l'OFS passe avant de basses préoccupations juridiques ou éthiques... et puis, le secret médical est apparemment une notion d'un autre âge, dépassée, inutile. Voilà!

\section{Ce projet est un scandale au regard de l'idée} de secret médical, une contradiction flagrante avec la loi, et une absurdité sur la plan de la surcharge administrative des cabinets.

De quoi s'agit-il? L'article 22a LAMal [1] prévoit la transmission de données des cabinets à l'OFS, aux fins de «surveiller l'application des dispositions de la [LAMal] relatives au caractère économique et à la qualité des prestations». Et les données requises sont entre autres «le nombre de patients et la structure de leur effectif, sous une forme anonyme».

Bon, cette loi est en vigueur depuis le $1^{\text {er }}$ janvier 2009 et il faudra bien finir par l'appliquer. Mais pas n'importe comment!

Car l'Office fédéral de la statistique s'obstine à vouloir, dans la mise en œuvre de cette disposition, recevoir toutes les informations sur tous les patients, sur toutes les consultations de tous les cabinets médicaux du pays - des données même pas vraiment anonymisées car elles doivent finalement per-

\section{La confiance envers le médecin est por- teuse du traitement et de la guérison.}

mettre de constituer pour toute personne en Suisse un «curriculum de santé». Ce projet est donc un vrai scandale au regard de l'idée de secret médical, une contradiction flagrante avec la loi qui ne demande que «le nombre de patients et la structure de leur effectif, sous une forme anonyme», et enfin une absurdité spectaculaire sur la plan de la surcharge administrative des cabinets.
Il est clair que la FMH, au nom tant du corps médical que des principes essentiels de la médecine, va s'opposer farouchement à ce projet MARS (tel est son nom!) de l'OFS. Nous n'accepterons simplement pas cette mise en fiches de la population, cette instrumentalisation du corps médical, cette menée illégale d'un Office fédéral.

\section{Un élément aussi central que la méde- cine, dans une société humaine, ne peut être impunément écarté ou dénaturé.}

Ceci dit, cette boulimie de l'Administration fédérale peut aussi être l'occasion d'expliciter une fois encore pourquoi le secret médical est réellement important.

Il est clair que les possibilités technologiques qui se développent actuellement permettent de presque tout faire, en matière de stockage de données et de surveillance des individus - qu'ils soient d'ailleurs médecins ou patients, en l'occurrence. Ce n'est toutefois pas une raison pour tout faire, justement! Faut-il rappeler par exemple qu'aller aussi vite qu'on le peut sur la route, que faire tout ce que permettent les découvertes de la physique nucléaire, que toujours faire tout ce que permet la médecine, ce n'est pas très intelligent?

Chaque découverte, chaque avancée scientifique ou technologique, chaque nouvelle possibilité demande un moment de réflexion, de discernement. Vouloir tout faire «parce qu'on le peut» relève de l'infantilisme.

Et puis, second élément de réflexion plus spécifique, on ne peut oublier que dans la société humaine, la médecine a une histoire, une substance, une place qui n'est pas seulement faite de pilules et de suppositoires; la médecine est un élément central de toute société, de la nôtre aussi, avec un rôle tout à fait particulier, auquel le respect de la personne et la confidentialité, le secret, sont essentiels.

Evoquer ici le Serment d'Hippocrate est une banalité. Ce texte vieux de 2500 ans démontre cependant fort bien, dans sa partie consacrée au secret médical, que ce dernier n'est pas une lubie contemporaine, mais que la confiance envers le médecin, cette personne à qui l'on ne parle pas que de fièvre et de boutons mais aussi de soi, de sa vie, de ses questionnements et de ses errements, que cette confiance est en réalité porteuse du traitement et de la guérison.

Il nous appartient de défendre cette médecine-là... et donc de rappeler autant qu'il sera nécessaire à nos responsables politico-administratifs qu'un élément aussi central que la médecine, dans une société humaine, ne peut être impunément écarté ou dénaturé.

Dr Jacques de Haller, Président de la FMH

1 www.admin.ch/ch/f/rs/832_10/a22a.html 